\title{
Carte génique du lapin (Oryctolagus cuniculus L.) : synténie entre les gènes utéroglobine, lactate déshydrogénase A et phosphatase acide 2
}

\author{
J. GELLIN, M. DALENS, Geneviève ECHARD et F. HATEY \\ I.N.R.A., Centre de Recherches de Toulouse, Laboratoire de Génétique cellulaire \\ Chemin de Borde-Rouge, B.P. 12, F 31320 Castanet-Tolosan
}

\begin{abstract}
Résumé
L'expression de quatorze enzymes (analyse électrophorétique) et la présence du gène de l'utéroglobine (hybridation moléculaire de l'ADN) ont été recherchées dans vingt-sept clones cellulaires hybrides lapin-hamster. Les résultats permettent de définir, chez le lapin, une nouvelle synténie, entre UGL 1 et LDHA-ACP 2.
\end{abstract}

Mots clés : Lapin, carte génique, utéroglobine, LDHA, ACP 2.

\author{
Summary \\ Rabbit gene mapping : uteroglobin, lactate dehydrogenase $A$, \\ acid phosphatase synteny
}

The expression of fourteen enzymes (electrophoresis analysis) and the presence of uteroglobin gene (DNA molecular hybridization) were investigated in twenty seven rabbithamster somatic cell hybrids. UGL 1 was found to be syntenic with LDHA and ACP 2 in rabbits.

Key words : Rabbit, gene mapping, uteroglobin, LDHA, ACP 2.

\section{Introduction}

La carte génique du lapin (revue générale : O'Brien, 1982) a tout d'abord progressé par des études de familles qui ont permis de définir neuf groupes de liaisons autosomaux. La perte aléatoire des chromosomes du lapin dans les hybrides cellulaires lapin-hamster permet d'analyser un plus grand nombre de caractères et, ainsi, de faire avancer la connaissance de la carte génique (Echard \& Gillois, 1979 ; Cianfriglia et al., 1979). Cinq autres groupes synténiques ont ainsi été décrits, dont l'un, de quatre 
gènes, sur le chromosome $\mathrm{X}$. Ces résultats ont été obtenus par l'analyse électrophorétique des protéines codées par ces gènes.

L'hybridation moléculaire de l'ADN des hybrides cellulaires avec des sondes d'ADN cloné permet de révéler la présence des gènes correspondants, que ceux-ci soient exprimés ou non (OWERBACH et al., 1980). Nous avons utilisé cette méthode pour mettre en évidence, dans les hybrides cellulaires, le gène de l'utéroglobine, protéine secrétée par l'endomètre de la lapine et dont la synthèse est induite par la progestérone (SAVOuRET et al., 1980).

Nos résultats démontrent que le gène de l'utéroglobine (UGL1) est synténique avec les gènes de la lactate déshydrogénase (LDHA) et de la phosphatase acide (ACP2).

\section{Matériels et méthodes}

Parmi les hybrides cellulaires lapin-hamster déjà décrits (EcHARd et al., 1981), nous avons sélectionné vingt-sept clones indépendants dérivant de trois lapins différents. Les extraits cellulaires (électrophorèse) et les préparations d'ADN (hybridation moléculaire) sont issus des cellules d'une même culture, donc au même stade de leur évolution. Nous avons analysé les enzymes suivantes : ACY (acylase), ENO (énolase), GPI (glucose phosphate isomérase), GSR (glutathion réductase) LDHA et B (lactate déshydrogénase A et B), MDH1 et 2 (malate déshycrogénase 1 et 2), NP (nucléoside phosphorylase), ACP2 (phosphatase acide 2), PGD (phosphoglucodéshydrogénase), PGM1 (phosphoglucomutase), PEPB (peptidase B), TPI (triose phosphate isomérase). Les techniques d'électrophorèse et de coloration spécifique des enzymes sont déjà décrites (EcharD et al., 1982).

L'ADN des cellules parentales et des cellules hybrides est purifié (Gross-BELLARD et al., 1973) et digéré par l'enzyme de restriction $\mathrm{EcoR}_{1}$. L'ADN du phage $\lambda$ digéré par l'enzyme de restriction Hind III est utilisé comme marqueur de taille. Après migration sur un gel d'agarose, l'ADN est transféré in situ sur une feuille de nitrocellulose (SoutherN, 1979). La sonde UGL1 dérive de la génothèque d'ADN de lapin réalisée par Maniatis et al. (1978). Cette sonde est constituée par un fragment de 18,4 kb (kilo base) d'ADN génomique inséré dans le phage Lambda Charon $4 \mathrm{~A}$. Le gène utéroglobine présent dans cette insertion a été caractérisé et étudié par ATGER et al. (1981). La sonde a été marquée au phosphore ¿2P par «nick-translation》 (RIGBY et al., 1977) à l'aide de deux nucléotides marqués (dCTP et TTP à $800 \mathrm{ci} / \mathrm{mmole}$, NEN). L'activité spécifique obtenue dépasse $10^{8} \mathrm{cpm} / \mu \mathrm{g}$. L'hybridation moléculaire s'effectue dans 50 p. 100 de formamide à $42^{\circ} \mathrm{C}$ pendant $16 \mathrm{~h}$. suivie d'une décontamination à $68^{\circ} \mathrm{C}$ (Southern, 1979). Les autoradiogrammes sont obtenus par une exposition des films pendant 4 jours à $-80^{\circ} \mathrm{C}$.

\section{Résultats et discussion}

Après digestion par $\mathrm{EcoR}_{1}$, l'ADN des cellules de hamster $\mathrm{wg}_{3} \mathrm{~h}$-cl.2 n hybride pas, dans nos conditions expérimentales, avec la sonde UGL1 (fig. 1). Par contre, 


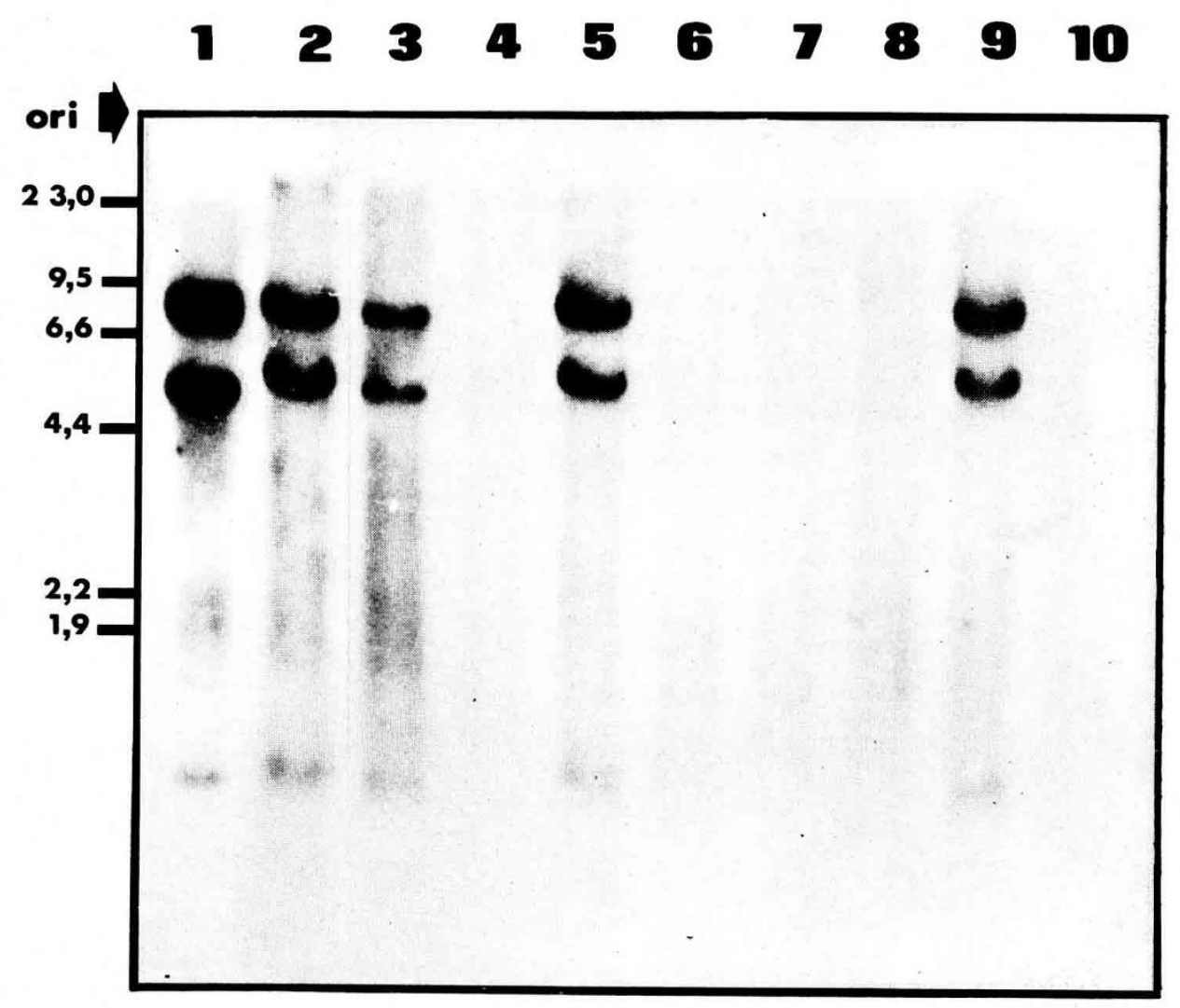

FIG. 1

Autoradiogramme obtenu après inybridation moléculaire de la sonde UGL 1 avec l'ADN parental ou l'ADN d'hybrides cellulaires lapin-hamster.

Autoradiogram obtained after molecular hybridization of the UGL 1 probe with DNA from either rabbit or hamster or rabbit $\times$ hamster cellular hybrids.

1) ADN de lapin.

Rabbit DNA.

2), 3), 5), 9) Hybrides cellulaires positifs.

Positive cellular hybrids.

4), 6), 7), 8) Hybrides cellulaires négatifs.

Negative cellular hybrids.

10) ADN de hamster $\mathrm{wg}_{3} \mathrm{~h}-\mathrm{cl}_{2}$.

Hamster DNA.

L'ADN du phage $\lambda$ digéré par l'enzyme de restriction Hind III a été utilisé comme marqueur de taille $(\mathrm{kb})$.

Hind III restriction enzyme digest of phage $\lambda$ DNA is used as size marker $(k b)$. 
l'ADN des cellules de lapin présente deux bandes majeures $(7,5$ et $5,5 \mathrm{~kb})$ et une bande plus faible d'environ $1 \mathrm{~kb}$. Selon les clones étudiés, l'ADN des cellules hybrides présente ou non l'image de l'ADN de lapin. Dans le premier cas, les clones ont conservé le chromosome qui porte le gène de l'utéroglobine de lapin et sont positifs. Dans le deuxième cas, les clones ont perdu le chromosome et sont négatifs. Nous avons constaté que douze clones sont UGL1-LDHA-ACP2 positifs et que douze clones sont UGL1-LDHA-ACP2 négatifs. Trois clones seulement sont discordants. Deux d'entre eux sont UGL1 négatifs et LDHA-ACP2 positifs et le troisième est UGL1-ACP2 négatif, LDHA positif (tabl. 1). En dehors de ces trois clones discordants, vraisemblablement dus à des cassures chromosomiques, il y a maintien ou perte simultanée de ces trois caractères. Ces résultats mettent en évidence une synténie UGL1-LDHAACP2. Aucune autre corrélation n'a été observée avec les autres enzymes analysées (ECHARD et al., 1982).

TABLEAU 1

Corrélation entre les marqueurs UGL1 - LDHA - ACP2.

Correlation between $U G L 1-L D H A-A C P 2$.

\begin{tabular}{|c|c|c|c|c|c|c|c|c|c|}
\hline \multirow{5}{*}{$\begin{array}{l}\text { Mar- } \\
\text { queur } 2\end{array}$} & \multicolumn{4}{|c|}{ Marqueur 1} & \multirow{5}{*}{ LDHA } & \multicolumn{4}{|c|}{ UGL1 } \\
\hline & & + & 一 & \multirow{2}{*}{$\ldots$} & & & + & - & \\
\hline & + & a & c & & & + & 12 & 3 & \multirow[b]{3}{*}{2} \\
\hline & 一 & b & d & \multirow[b]{2}{*}{$\mathbf{T}$} & & - & \multirow[t]{2}{*}{0} & \multirow[t]{2}{*}{12} & \\
\hline & & & & & & & & & \\
\hline \multirow{5}{*}{ ACP2 } & \multicolumn{4}{|c|}{ UGL1 } & \multirow{5}{*}{$\mathrm{ACP} 2$} & \multicolumn{4}{|c|}{ LDHA } \\
\hline & & + & 一 & & & & + & 一 & \\
\hline & + & 12 & 2 & & & + & 14 & 0 & \\
\hline & 一 & 0 & 13 & & & - & 1 & 12 & \\
\hline & & & & 27 & & & & & 27 \\
\hline
\end{tabular}

T - Nombre total de clones cellulaires hybrides. Number of cellular hybrid clones.

a - Nombre de clones cellulaires hybrides positifs pour les deux marqueurs. Number of positive cellular hybrid clones for the two markers.

b - Nombre de clones cellulaires hybrides positifs pour le marqueur 1 , et négatifs pour le marqueur 2.

Number of positive cellular hybrid clones for marker 1 , and negative for marker 2.

c - Nombre de clones cellulaires hybrides positits pour le marqueur 2, et négatifs pour le marqueur 1.

Number of positive cellular hybrid clones for marker 2, and negative for marker 1.

d - Nombre de clones cellulaires hybrides négatifs pour les deux marqueurs. Number of negative cellular hybrid clones for the two markers. 
LDHA et ACP2 sont synténiques chez l'homme (chromosome HSA11), chez le chimpanzé (PTR9), le singe rhésus (MML11), mais non synténiques chez la souris (LDHA sur le chromosome MMU7 et ACP2 sur le chromosome MMU2). Il n'est fait aucune mention de la localisation du gène utéroglobine dans la revue générale de O'Brien (1982) portant sur l'ensemble des espèces. Nos résultats sur le lapin représentent la première synténie décrite concernant le gène de l'utéroglobine.

Soulie et al. (1982) ont assigné le gène LDHA au chromosome 1 du lapin (OCU1). Certains de nos hybrides cellulaires ont peu de chromosomes (ECHARD et al., 1981) et l'étude du caryotype de ces hybrides, ainsi que celle des sous-clones en cours de préparation, devraient nous permettre de confirmer ou non cette assignation.

Les techniques utilisées dans cet article permettent donc l'étude de gènes non exprimés de façon constitutive dans les cellules et augmentent le nombre de marqueurs utilisables pour l'établissement de la carte génique. Elles permettent également d'aborder l'étude de gènes intervenant dans des fonctions physiologiques complexes sous contrôle hormonal.

Reçu le 5 avril 1983.

Accepté le 17 juin 1893.

\section{Remerciements}

Les auteurs remercient M. Gillois, directeur du laboratoire de Génétique cellulaire, pour son aide et ses encouragements ainsi que M. ATGER (Groupe de Recherches sur la Biochimie Endocrinienne et la Reproduction, Unité 135 de l'I.N.S.E.R.M.) qui a fourni l'ADN de la sonde UGL 1 .

\section{Références bibliographiques}

Atger M., Atger P., Tiollais P., Milgrom E., 1981. Cloning of rabbit genomic fragments containing the uteroglobin gene. J. Biol. Chem., 256, 5970-5972.

Cianfriglia M., Battistuzzi G., Meo T., Miggliano V.C., 1979. Assignment of the gene for LDHB to rabbit chromosome 14 using mouse $\mathrm{x}$ rabbit somatic cell hybrids. Proceedings of the Vth International Conference on Human Gene Mapping, Edinburg, July 9-13, 1979. Cytogenet. Cell Genet., 25, 141-142.

ECHARD G., GILloIS M., 1979. G6PD-PGK-GLA-HPRT synteny in the rabbit (Oryctolagus caniculus $\mathrm{L}$.). Proceedings of the Vth International Conference on Human Gene Mapping, Edinburg, July 9-13, 1979. Cytogenet. Cell Genet., 25, 148-149.

Echard G., Gellin J., Benne F., Gillois M., 1981. The gene map of the rabbit (Oryctolagus cuniculus L.). I - Synteny between the rabbit gene loci coding for HPRT, PGK, G6PD and GLA. Their localization on the X-chromosome. Cytogenet. Cell Genet., 29, 176-183.

Echard G., Gellin J., Benne F., Gillors M., 1982. The gene map of the rabbit (Oryctolagus cuniculus L.). II - Analysis of the segregation of 11 enzymes in rabbit $\times$ hamster somatic cell hybrids : two syntenic groups, LDHB-TPI and LDHA-ACP 2. Cytogenet. Cell Genet., 34, 289-295. 
Gross-Bellard M., Oudet P., Chambon P., 1973. Isolation of high-molecular-weight DNA from mammalian cells. Eur. J. Biochem., 36, 32-38.

Maniatis T., Hardison R.C., Lacy E., Lauer J., O'Connell C., Quon D., 1978. The isolation of structural genes from librairies of eucaryotic DNA. Cell, 15, 687-701.

O'BRIEN S.J., 1982. A compilation of linkage and restriction maps of genetically studied organisms. Genet. Maps, 2, 277-359.

OWerbach D., Rutter W.J., Martial J.A., Baxter J.D., Shows J.B., 1980. Genes for growth hormone chorionic somatomammotropin and growth hormone-like gene on chromosome 17 in humans. Science, 209, 289-292.

Rigby P.W.J., Bieckmann M., Rhodes C., Berg P., 1977. Labeling deoxyribonucleic acid to high specific activity in vitro by nick translation with DNA polymerase I. J. Mol. Biol., 113, 237-251.

Savouret J.F., Loosfelt H., Atger M., Milgrom E., 1980. Differential hormonal control of a messenger RNA in two tissues : uteroglobin mRNA in the lung and the endometrium. J. Biol. Chem., 225, 4131-4136.

Soulie J., DE Grouchy J., 1982. Of rabbit and man : comparative gene mapping. Hum. Genet., 60, 172-175.

SOUTHERn E., 1979. Gel electrophoresis of restriction fragments. Methods Enzymol., 68, $152-176$. 